\title{
Rac1 at the crossroad of actin dynamics and neuroinflammation in Amyotrophic Lateral Sclerosis
}

\author{
Nadia D'Ambrosi ${ }^{1}$, Simona Rossi ${ }^{2,3}$, Valeria Gerbino ${ }^{3,4}$ and Mauro Cozzolino ${ }^{2}$ * \\ 1 Institute of Anatomy and Cell Biology, Università Cattolica del Sacro Cuore, Rome, Italy \\ ${ }^{2}$ National Research Council, Institute of Translational Pharmacology, Rome, Italy \\ ${ }^{3}$ Fondazione Santa Lucia IRCCS, Rome, Italy \\ ${ }^{4}$ Department of Biology, Università di Roma Tor Vergata, Rome, Italy
}

\section{Edited by:}

Trisha Stankiewicz, University of

Denver, USA

\section{Reviewed by:}

Muzamil Ahmad, Indian Institute of Integrative Medicine, India

Ping Liu, University of Connecticut Health Center, USA

*Correspondence:

Mauro Cozzolino, National Research Council, Institute of Translational Pharmacology, Via del Fosso del Cavaliere 100, 00133 Rome, Italy e-mail:mauro.cozzolino@ift.cnr.it
Rac1 is a major player of the Rho family of small GTPases that controls multiple cell signaling pathways, such as the organization of cytoskeleton (including adhesion and motility), cell proliferation, apoptosis and activation of immune cells. In the nervous system, in particular, Rac1 GTPase plays a key regulatory function of both actin and microtubule cytoskeletal dynamics and thus it is central to axonal growth and stability, as well as dendrite and spine structural plasticity. Rac1 is also a crucial regulator of NADPH-dependent membrane oxidase (NOX), a prominent source of reactive oxygen species (ROS), thus having a central role in the inflammatory response and neurotoxicity mediated by microglia cells in the nervous system. As such, alterations in Rac1 activity might well be involved in the processes that give rise to Amyotrophic Lateral Sclerosis (ALS), a complex syndrome where cytoskeletal disturbances in motor neurons and redox alterations in the inflammatory compartment play pivotal and synergic roles in the final disease outcomes. Here we will discuss the genetic and mechanistic evidence indicating the relevance of Rac1 dysregulation in the pathogenesis of ALS.

Keywords: Amyotrophic Lateral Sclerosis (ALS), Rac1, neuroinflammation, motor neurons, NOX, microglia, reactive oxygen species, spinal muscular atrophy (SMA)

\section{INTRODUCTION}

Degeneration of motor neurons in the brain cortex, brainstem and spinal cord of patients is the most recognized feature of Amyotrophic Lateral Sclerosis (ALS). Since the discovery of the first gene associated to familial forms of ALS, Sod1, many fundamental achievements in clinical, genetic, and basic biology processes related to ALS have profoundly improved our knowledge of the disease (Cozzolino et al., 2012). Indeed, while it has long been thinking that ALS was a disease highly specific for motor neurons, with a low albeit significant incidence of genetics limited to familial forms, it is now widely accepted that ALS is a complex syndrome that involves a wide range of different tissues and cell types, including muscle cells, astrocytes, microglia, oligodendrocytes. Further, it is clear that genes have a profound impact on the overall disease pathogenesis, even in apparently sporadic forms (Renton et al., 2014). To make things more intricate, many of the recently identified gene mutations that are definitely involved in familial (and to some degrees in sporadic) forms of ALS are also responsible of, or associated to diseases that do not primarily manifest as motor neuron diseases, ranging from Fronto Temporal Dementia (FTD) to Huntington Disease Phenocopies, putting in the spotlight the concept that ALS is part of a more widespread and complex disease process (Cooper-Knock et al., 2014).
Although different mechanisms have been invoked to play a role in the pathogenesis of ALS (Cozzolino et al., 2012), RNA metabolism has emerged as a fundamental aspect of disease determination. Two different lines of reason support this conclusion. First, recent genetic evidence pinpointed at least three different genes whose mutations, together accounting for nearly $50 \%$ of familial ALS cases, are likely responsible for diseaserelevant alterations in RNA control. These genes include TARDBP (Neumann et al., 2006), FUS/TLS (Vance et al., 2009), and c9orf72 (DeJesus-Hernandez et al., 2011; Renton et al., 2011). In all cases, mutations in these genes are believed to alter the control of RNA processing, including mRNA transcription, splicing, translation and transport (Ling et al., 2013), although the particular step of RNA metabolism that is affected in ALS is still to be ascertained.

Second, analogies with another Motor Neuron Disease (MND), namely Spinal Muscular Atrophy (SMA), sustain the idea that post-transcriptional gene regulation is profoundly affected in MND, including ALS. Indeed, SMA is caused by a decreased availability of the Survival Motor Neuron protein $(\mathrm{SMN})$, due to gene mutations or deletions in the smn1 gene, and this leads to an early degeneration of lower motor neurons in children. SMN is known to play a crucial function both in the biogenesis and localization of small ribonucleoprotein particles (snRNPs), the core modules of the splicing machinery 
(Burghes and Beattie, 2009), and in the transport along neuronal processes of cytosolic mRNAs-containing granules (Rage et al., 2013), an essential process that guarantees local protein synthesis (Jung et al., 2014). This is particularly important in, although not limited to, neurons, where local mRNA translation sustains axon elongation and maintenance, as well as synapses formation and plasticity (Jung et al., 2012). Thus, in the complete absence of SMN, cells and animals die (Schrank et al., 1997), to testify the widespread and essential role of SMN, while the shortage of SMN protein is particularly harmful to neurons. Although the reasons why a decreased availability of SMN activates a very selective process that leads to the specific degeneration of lower motor neurons is far from being clear, evidence exist that mys-functioning in SMN-regulated processes might be shared by ALS (Yamazaki et al., 2012; Gerbino et al., 2013; Groen et al., 2013; Turner et al., 2014), further indicating that motor neurons are particularly vulnerable to disturbed RNA metabolism.

\section{EVIDENCE POINTING TO DYS-REGULATION OF ACTIN DYNAMICS IN MNDs: RHO GTPases AND MOTOR NEURONS}

The overlap between ALS and SMA is not however restricted to RNA control. Indeed, ALS and SMA share actin dynamics disturbances as a central route for motor neuron degeneration. In particular, the regulation of actin dynamics at the synapse, which is accomplished by a plethora of actin-binding/-regulating proteins is crucial for the proper function and maturation of neuromuscular junctions (NMJs), that are supposed to be a primary, early target of both ALS and SMA (Fischer et al., 2004; Kariya et al., 2008).

Both $\beta$-actin mRNA and protein are significantly reduced in distal axons and growth cones of SMA motor neurons, thus establishing a link between actin perturbations to the degeneration of NMJs in SMA (Rossoll et al., 2003). Similarly, overexpression of SMN in cultured neuronal cells promotes neurite outgrowth, while downregulation has an opposite effect (van Bergeijk et al., 2007). This is the consequence of changes in the ratio between F- and G-actin, further indicating that SMN regulates actin dynamics. Actually, the overexpression in SMA motor neurons of the actin-binding protein plastin 3, a genetic modifier for SMA (Oprea et al., 2008), rescues defects due to decreased SMN (Ackermann et al., 2013). Microfilament dynamics are also apparently mediated by profilins (Birbach, 2008) and SMN was shown, in particular, to bind and regulate the expression of profilin $2 \mathrm{a}$, which is known to promote actin polymerization (Nölle et al., 2011). Profilin2a is a key player in the Rho-kinase (ROCK) pathway, and loss of SMN results in an increased activity of the small GTPase RhoA, and an increased binding of profilin2a to ROCK, a direct downstream effector of RhoA. RhoA-mediated activation of ROCK then leads to a consequent ROCK-dependent hyperphosphorylation of profilin 2 a which in this form eventually represses neurite outgrowth in neuronal cells (Bowerman et al., 2010; Nölle et al., 2011). The functional relevance of this pathway is clearly demonstrated by the observation that treating SMA mice with ROCK inhibitors strongly improves the maturation of the NMJs, increases muscle fiber size and prolongs their survival (Bowerman et al., 2010, 2012).
Motor neuron loss is also delayed in experimental models of ALS by fasudil, a clinical approved ROCK inhibitor (Takata et al., 2013). These results thus indicate that a similar pattern of deregulation affecting the RhoA/ROCK/profilin pathway might be operating in the degenerating motor neurons in ALS. Mutations within the profilin 1 (PFN1) gene have been identified in late 2012 to cause ALS in two large families (Wu et al., 2012). PFN1 is crucial for the conversion of monomeric to filamentous actin, and ALS-linked PFN1 mutations were shown to reduce the ability of PFN1 to bind actin and to induce a decrease in the ratio of $F / G$ actin. This eventually leads to inhibition of neurite outgrowth and alteration in growth cone morphology, all features clearly reminiscent of actin dynamics disturbances. Although later mutational screening in worldwide ALS populations established that PFN1 gene mutations are a very rare cause of familial ALS (van Blitterswijk et al., 2013), these findings, together with the association of familial ALS to rare mutation in cytoskeleton-regulating genes such as neurofilament heavy polypeptide, peripherin and dynactin provide a considerable genetic basis to disruption of actin cytoskeleton as a possible pathogenic mechanism in motor neuron degeneration (Renton et al., 2014).

As central regulators of actin dynamics, Rho family of small GTPases, with RhoA, Rac1 and cdc42 the most studied members, are natural candidates to play a role in these processes. A growing body of evidence suggests, in particular, that inhibition of Racl mediates the process of motor neuron degeneration in ALS. Mutations in ALS2 were described to be responsible for a recessive form of juvenile-onset ALS (Yang et al., 2001), although later studies have suggested that ALS2-induced disease phenotypes are more related to slowly progressive, juvenile forms of hereditary spastic paraplegia (Panzeri et al., 2006). Nonetheless, ALS2 encodes for the protein alsin, which has been characterized as a guanine nucleotide exchange factor (GEF) for Rho family of small GTPases, including Rac1, and mutation in alsin are expected to affect GEF activity of this protein (Yang et al., 2001; Topp et al., 2004). In line with its activatory function, alsin knockdown inhibits axon growth and induces cell death in cultured motor neurons, further indicating the importance of cytoskeletal balance in motor neuron health. Importantly, cell death and cytoskeletal alterations are obtained in the same cells by overexpression of a dominant-negative Racl mutant, while a constitutively active Racl mutant is able to inhibit it (Jacquier et al., 2006). Moreover, the apoptotic phenotypes induced in NSC34 motor neuron-like cells by overexpression of mutSOD1 are inhibited by co-expression of alsin, and this neuroprotective activity is completely abolished by decreasing endogenous Rac1 with siRNA (Kanekura et al., 2005), indicating that Racl acts downstream of alsin. Overall, these observations clearly indicate that disruption of Racl GTPase function is a contributing event in motor neuron degeneration in ALS. This conclusion is further supported by findings from experiments in cultured neuronal cells depleted of TDP43. Indeed, TDP43 knockdown in neuronal cells inhibits neurite outgrowth and induces cell death, as a consequence of decreased membrane association and inactivation of RhoA, Rac1, and cdc42 Rho GTPases (Iguchi et al., 2009). However, it has also been shown that TDP43 knockdown 
in hippocampal neurons is able to repress Racl expression at a translational level, and this causes a significant increase in the density of dendritic spines (Majumder et al., 2012), thus further indicating that the outcome of Rho GTPases activity is indeed a unique feature depending on the specific neuronal cell subpopulation.

All these results point to Rac1-dependent cytoskeletal dysregulation as a key determinant of motor neuron degeneration. However, beyond their characterized function in actin dynamics, Rho GTPase signaling is also involved in other important cellular processes (Linseman and Loucks, 2008) that might be altered in ALS. An important link between Racl and motoneuronal cell viability was provided by the observation that the overexpression of mutSOD1 proteins decreases the levels of active, GTP-bound form of Racl and induces apoptosis in SH-SY5Y neuronal cell lines (Pesaresi et al., 2011). Importantly, the overexpression of a constitutive active form of Rac1 (V12) fully protects SH-SY5Y cells from apoptosis, whereas a dominantnegative, inactive form of mutant of Racl (N17) is per se sufficient to induce apoptotic cell death. Thus, Rac1 inhibition is anticipated to have a critical role in motor neurons demise in ALS. Yet, a direct evidence that this is occurring in motor neurons in vivo, has not been reported so far. Interestingly, the inhibition of Rac1 by mutSOD1s is strictly dependent on the activation of a signaling pathway which originates from mitochondria and is mediated by the p66KDa isoform of the adaptor protein Shc (p66Shc): when mitochondrial damage is prevented by p66Shc functional inhibition, Racl activity is rescued, and cell survival recovered. Thus, damage to mitochondria, which represent a well recognized, early sign of motor neuron degeneration in ALS, might represent the trigger of a pathological cascade involving Racl dysregulation, eventually leading to both actin cytoskeleton disturbances and loss of cell viability. In addition, these data point to mitochondria-derived reactive oxygen species (ROS) as mediators of Racl inhibition. Many types of hints are indeed accumulating, which indicate that Rho family GTPases are a redox sensitive family of proteins (Mitchell et al., 2013). This is of crucial importance in ALS, a pathological condition that is associated to critical alterations in the redox balance which exert detrimental effects not only at the level of NMJ (Pollari et al., 2014), but also in the inflammatory compartment where ROS-mediated microglial cells activation plays a fundamental role in ALS disease progression (Boillée et al., 2006).

\section{Rac1 AS A MAJOR PLAYER IN INFLAMMATION IN ALS}

The neuroinflammatory process is a feature common to all ALS forms, either sporadic or familial, and in the latter case all ALScausative genes exert a comparable activation of the glial compartment, despite their specific function (Philips and Robberecht, 2011; Rizzo et al., 2014). It is commonly believed that the neuroinflammatory reaction played in particular by microglia, the resident immune cells of the CNS, exerts a protective function at early stages of the pathology that is switched into a detrimental one as the disease progresses (Appel et al., 2011). Accordingly, microglia cells producing trophic, beneficial effects are said to belong to the M2 phenotype, whilst the toxic phenotype is defined as M1. In response to stimuli derived from damaged motor neurons and from autocrine/paracrine glial signaling, M1 microglia produces and releases ROS and pro-inflammatory cytokines that are responsible for a self-propagating cycle of progressive and irreversible motor neuron death (Henkel et al., 2009). The inhibition of such damaging pro-inflammatory process is therefore a main field in ALS research and one target of interest in this context is NOX.

In particular, NOX2 (gp91phox) is a major source of superoxide by phagocytic cells (Bedard and Krause, 2007), and is the predominant isoform found in microglia, astrocytes and neurons (Sorce and Krause, 2009), where its activation results in a neurotoxic outcome (Marchetto et al., 2008; Liao et al., 2012). NOX2 expression is upregulated both in familial and sporadic ALS patients and in different ALS-animal models, and NOX2 inhibition by genetic ablation in SOD1-G93A mice significantly slows disease progression and extends survival (Wu et al., 2006; Marden et al., 2007). Pharmacological inhibition of NOX2 by apocynin was shown to protect motor neurons from mutant SOD1 toxicity in culture (Harraz et al., 2008; Marchetto et al., 2008; Trumbull et al., 2012), but gave contrasting results when administered to SOD1-G93A mice (Harraz et al., 2008; Trumbull et al., 2012). Anyway, NOX2 is considered one of the main markers of M1-toxic microglia. NOX2 consists of the integral membrane subunits gp $91^{\text {Phox }}$ and $\mathrm{p} 22^{\text {Phox }}$, which are permanently associated with each other to form the catalytic flavocytochrome $b_{558}$ core (cyt $b_{558}$ ), the cytosolic subunits $\mathrm{p} 40^{\text {Phox }}$, $\mathrm{p} 47^{\text {Phox }}, \mathrm{p} 67^{\text {Phox }}$ and Rac1. Upon activation, the cytosolic factors translocate to membranes to interact with $\mathrm{cytb}_{558}$, through which electrons from NADPH are transported to reduce molecular oxygen to superoxide. Active, GTP-bound Rac1 acts both as an adaptor, to ensure correct positioning of $\mathrm{p} 67^{\text {Phox }}$ toward $c y t b_{558}$, and as a player in the electron-transfer reaction (Bedard and Krause, 2007).

In ALS, uncontrolled Racl activation represents a straight link between disease-causing mutSOD1s and NOX2-mediated ROS production by glial cells. It was in fact clearly demonstrated that SOD1 directly binds to Racl in a redox-sensitive manner, influencing the rate of intrinsic GTP hydrolysis, and thus of Racl activity: in reducing conditions SOD1 binds to Racl and stimulates its activity; conversely, in oxidizing conditions SOD1 dissociates from Rac1, favoring GTP to GDP exchange and thus eventually silencing Rac1-dependent activity of NOX2. This mechanism is found dysregulated in glial cells by different SOD1 mutants, that are less sensitive to redox uncoupling, in particular in a subpopulation of early endosomes containing NOX2, called redoxosomes (Harraz et al., 2008; Li et al., 2011). As a matter of fact, expression of mutSOD1s in glial cells elevates the levels of GTP-bound Rac1 (Harraz et al., 2008), that are also found dramatically increased in microglia derived from SOD1G93A mice (Apolloni et al., 2013). ROS generated by the Rac1NOX2 axis are then responsible for $\mathrm{TNF} \alpha$ secretion, autocrine and paracrine induction of the pro-inflammatory transcription factor NFKB in co-cultured neurons and neurotoxicity (Li et al., 2011). Substantial raise of active Racl is also found in spinal cord from SOD1-G93A mice, with consequent hyperactivation of NOX2 (Harraz et al., 2008). 
The overactivation of Rac1/NOX2 pathway in vivo could be also the resultant of the action of different pro-inflammatory molecules, such as extracellular ATP. This nucleotide is an activator of microglia response through purinergic receptors and it is supposed to be increasingly released during neuronal damage. In ALS, the P2X7 receptor preferential agonist $2^{\prime}-3^{\prime}$-O-(benzoylbenzoyl) ATP, enhances the neurotoxic potential of SOD1-G93A microglia and increases the production of ROS through the recruitment of Rac1 and NOX2. Conversely, different P2X7 antagonists restore ROS production to basal levels decreasing the amount of Rac1-GTP and inhibiting NOX2 activity (Apolloni et al., 2013).

A role for Racl in ALS neuroinflammation is also suggested by the interplay between alsin and SOD1-G93A in influencing the dynamics of ROS production in glial cells. Alsin is known to bind three components of the redoxosome: it possesses RhoGEF domains for Rac1 and Rab5 and it also interacts with SOD1. When coexpressed with SOD1-G93A, alsin was shown to attenuate SOD1-dependent Racl activation, ROS generation by $\mathrm{NOX} 2, \mathrm{NF} \kappa \mathrm{B}$ induction, $\mathrm{TNF} \alpha$ secretion and to protect neurons from toxicity in co-culture studies (Li et al., 2011). This protective effect is in line with the detrimental one exerted by alsin knockdown in motor neurons (Jacquier et al., 2006) and appears to be mediated by the ability of alsin to decrease Racl activation in the presence of SOD1-G93A. These findings suggest that independent ALS disease-associated genes may converge on the same pathogenic pathway, i.e., the Rac1/NOX2 ROS-generating mechanism.

\section{THERAPEUTIC PERSPECTIVES TARGETING Rac1}

In this review, we have shown how cytoskeletal dynamics alterations in motor neurons as well as neuroinflammatory events in microglia underlie the pathogenesis of ALS and other MNDs, and how Racl GTPase might be involved in both processes. Although many gaps exist in our knowledge about the mechanistic aspects of these processes, Rac1 should be considered as a valuable target to interfere with the disease development.

Like other Rho GTPases, Racl represents a hub that responds to different signaling networks and in turn results in different, even contrasting outcomes by interacting with many diverse downstream effectors, that might well be different depending on the cell type as well as the stimulus involved. Thus, the apparently contradictory evidence about the activity of Racl in ALS motor neurons and microglia should not be regarded as surprising. Yet, the antagonistic effects that Racl targeting might have on the motor neurons as opposed to the inflammatory compartments poses some questions about how to devise an effective strategy, that will need to be answered in the near future. Nonetheless, a significant body of studies performed in contexts where aberrant Rac1 signaling is associated to alterations in neuronal structural plasticity and actin cytoskeleton dynamics indicates that the modulation of Racl-dependent signaling pathways could be effective to protect neuronal degeneration (Lorenzetto et al., 2013; Tönges et al., 2014; Travaglione et al., 2014). Of course, an accurate preclinical evaluation in animal models of ALS, that are widely available, will help answering these questions.

\section{ACKNOWLEDGMENTS}

Mauro Cozzolino is funded by Ministry of Health (RF-20102309849). Nadia D’Ambrosi is funded by UCSC (linea D1).

\section{REFERENCES}

Ackermann, B., Kröber, S., Torres-Benito, L., Borgmann, A., Peters, M., Hosseini Barkooie, S. M., et al. (2013). Plastin 3 ameliorates spinal muscular atrophy via delayed axon pruning and improves neuromuscular junction functionality. Hum. Mol. Genet. 22, 1328-1347. doi: 10.1093/hmg/dds540

Apolloni, S., Parisi, C., Pesaresi, M. G., Rossi, S., Carri, M. T., Cozzolino, M., et al. (2013). The NADPH oxidase pathway is dysregulated by the P2X7 receptor in the SOD1-G93 A microglia model of amyotrophic lateral sclerosis. J. Immunol. 190, 5187-5195. doi: 10.4049/jimmunol.1203262

Appel, S. H., Zhao, W., Beers, D. R., and Henkel, J. S. (2011). The microglialmotoneuron dialogue in ALS. Acta Myol. 30, 4-8.

Bedard, K., and Krause, K. H. (2007). The NOX family of ROS-generating NADPH oxidases: physiology and pathophysiology. Physiol. Rev. 87, 245-313. doi: 10. 1152/physrev.00044.2005

Birbach, A. (2008). Profilin, a multi-modal regulator of neuronal plasticity. Bioessays 30, 994-1002. doi: 10.1002/bies.20822

Boillée, S., Vande Velde, C., and Cleveland, D. W. (2006). ALS: a disease of motor neurons and their nonneuronal neighbors. Neuron 52, 39-59. doi: 10.1016/j. neuron.2006.09.018

Bowerman, M., Beauvais, A., Anderson, C. L., and Kothary, R. (2010). Rho-kinase inactivation prolongs survival of an intermediate SMA mouse model. Hum. Mol. Genet. 19, 1468-1478. doi: 10.1093/hmg/ddq021

Bowerman, M., Murray, L. M., Boyer, J. G., Anderson, C. L., and Kothary, R. (2012). Fasudil improves survival and promotes skeletal muscle development in a mouse model of spinal muscular atrophy. BMC Med. 10:24. doi: 10.1186/1741-7015$10-24$

Burghes, A. H., and Beattie, C. E. (2009). Spinal muscular atrophy: why do low levels of survival motor neuron protein make motor neurons sick? Nat. Rev. Neurosci. 10, 597-609. doi: 10.1038/nrn2670

Cooper-Knock, J., Shaw, P. J., and Kirby, J. (2014). The widening spectrum of C9ORF72-related disease; genotype/phenotype correlations and potential modifiers of clinical phenotype. Acta Neuropathol. 127, 333-345. doi: 10. 1007/s00401-014-1251-9

Cozzolino, M., Pesaresi, M. G., Gerbino, V., Grosskreutz, J., and Carri, M. T. (2012). Amyotrophic lateral sclerosis: new insights into underlying molecular mechanisms and opportunities for therapeutic intervention. Antioxid. Redox Signal. 17, 1277-1330. doi: 10.1089/ars.2011.4328

DeJesus-Hernandez, M., Mackenzie, I. R., Boeve, B. F., Boxer, A. L., Baker, M., Rutherford, N. J., et al. (2011). Expanded GGGGCC hexanucleotide repeat in noncoding region of C9ORF72 causes chromosome 9p-linked FTD and ALS. Neuron 72, 245-256. doi: 10.1016/j.neuron.2011.09.011

Fischer, L. R., Culver, D. G., Tennant, P., Davis, A. A., Wang, M., CastellanoSanchez, A., et al. (2004). Amyotrophic lateral sclerosis is a distal axonopathy: evidence in mice and man. Exp. Neurol. 185, 232-240. doi: 10.1016/j.expneurol. 2003.10.004

Gerbino, V., Carri, M. T., Cozzolino, M., and Achsel, T. (2013). Mislocalised FUS mutants stall spliceosomal snRNPs in the cytoplasm. Neurobiol. Dis. 55, 120128. doi: 10.1016/j.nbd.2013.03.003

Groen, E. J., Fumoto, K., Blokhuis, A. M., Engelen-Lee, J., Zhou, Y., van den Heuvel, D. M., et al. (2013). ALS-associated mutations in FUS disrupt the axonal distribution and function of SMN. Hum. Mol. Genet. 22, 3690-3704. doi: 10. 1093/hmg/ddt222

Harraz, M. M., Marden, J. J., Zhou, W., Zhang, Y., Williams, A., Sharov, V. S., et al. (2008). SOD1 mutations disrupt redox-sensitive Rac regulation of NADPH oxidase in a familial ALS model. J. Clin. Invest. 118, 659-670. doi: 10.1172/JCI34060

Henkel, J. S., Beers, D. R., Zhao, W., and Appel, S. H. (2009). Microglia in ALS: the good, the bad and the resting. J. Neuroimmune Pharmacol. 4, 389-398. doi: 10. 1007/s11481-009-9171-5

Iguchi, Y., Katsuno, M., Niwa, J., Yamada, S., Sone, J., Waza, M., et al. (2009). TDP43 depletion induces neuronal cell damage through dysregulation of Rho family GTPases. J. Biol. Chem. 284, 22059-22066. doi: 10.1074/jbc.M109.012195

Jacquier, A., Buhler, E., Schafer, M. K., Bohl, D., Blanchard, S., Beclin, C., et al. (2006). Alsin/Racl signaling controls survival and growth of spinal motoneurons. Ann. Neurol. 60, 105-117. doi: 10.1002/ana.20886 
Jung, H., Gkogkas, C. G., Sonenberg, N., and Holt, C. E. (2014). Remote control of gene function by local translation. Cell 157, 26-40. doi: 10.1016/j.cell.2014. 03.005

Jung, H., Yoon, B. C., and Holt, C. E. (2012). Axonal mRNA localization and local protein synthesis in nervous system assembly, maintenance and repair. Nat. Rev. Neurosci. 13, 308-324. doi: 10.1038/nrn3210

Kanekura, K., Hashimoto, Y., Kita, Y., Sasabe, J., Aiso, S., Nishimoto, I., et al. (2005). A Rac1/phosphatidylinositol 3-kinase/Akt3 anti-apoptotic pathway, triggered by AlsinLF, the product of the ALS2 gene, antagonizes $\mathrm{Cu} / \mathrm{Zn}$-superoxide dismutase (SOD1) mutant-induced motoneuronal cell death. J. Biol. Chem. 280, 45324543. doi: 10.1074/jbc.m410508200

Kariya, S., Park, G. H., Maeno-Hikichi, Y., Leykekhman, O., Lutz, C., Arkovitz, M. S., et al. (2008). Reduced SMN protein impairs maturation of the neuromuscular junctions in mouse models of spinal muscular atrophy. Hum. Mol. Genet. 17, 2552-2569. doi: 10.1093/hmg/ddn156

Li, Q., Spencer, N. Y., Pantazis, N. J., and Engelhardt, J. F. (2011). Alsin and SOD1(G93A) proteins regulate endosomal reactive oxygen species production by glial cells and proinflammatory pathways responsible for neurotoxicity. $J$. Biol. Chem. 286, 40151-40162. doi: 10.1074/jbc.M111.279711

Liao, B., Zhao, W., Beers, D. R., Henkel, J. S., and Appel, S. H. (2012). Transformation from a neuroprotective to a neurotoxic microglial phenotype in a mouse model of ALS. Exp. Neurol. 237, 147-152. doi: 10.1016/j.expneurol.2012.06.011

Ling, S. C., Polymenidou, M., and Cleveland, D. W. (2013). Converging mechanisms in ALS and FTD: disrupted RNA and protein homeostasis. Neuron 79, 416-438. doi: 10.1016/j.neuron.2013.07.033

Linseman, D. A., and Loucks, F. A. (2008). Diverse roles of Rho family GTPases in neuronal development, survival and death. Front. Biosci. 13, 657-676. doi: 10. $2741 / 2710$

Lorenzetto, E., Ettorre, M., Pontelli, V., Bolomini-Vittori, M., Bolognin, S., Zorzan, S., et al. (2013). Racl selective activation improves retina ganglion cell survival and regeneration. PLoS One 8:e64350. doi: 10.1371/journal.pone.0064350

Majumder, P., Chen, Y. T., Bose, J. K., Wu, C. C., Cheng, W. C., Cheng, S. J., et al. (2012). TDP-43 regulates the mammalian spinogenesis through translational repression of Rac1. Acta Neuropathol. 124, 231-245. doi: 10.1007/s00401-0121006-4

Marchetto, M. C., Muotri, A. R., Mu, Y., Smith, A. M., Cezar, G. G., and Gage, F. H. (2008). Non-cell-autonomous effect of human SOD1 G37R astrocytes on motor neurons derived from human embryonic stem cells. Cell Stem Cell 3, 649-657. doi: 10.1016/j.stem.2008.10.001

Marden, J. J., Harraz, M. M., Williams, A. J., Nelson, K., Luo, M., Paulson, H., et al. (2007). Redox modifier genes in amyotrophic lateral sclerosis in mice. J. Clin. Invest. 117, 2913-2919. doi: 10.1172/jci31265

Mitchell, L., Hobbs, G. A., Aghajanian, A., and Campbell, S. L. (2013). Redox regulation of Ras and Rho GTPases: mechanism and function. Antioxid. Redox Signal. 18, 250-258. doi: 10.1089/ars.2012.4687

Neumann, M., Sampathu, D. M., Kwong, L. K., Truax, A. C., Micsenyi, M. C., Chou, T. T., et al. (2006). Ubiquitinated TDP-43 in frontotemporal lobar degeneration and amyotrophic lateral sclerosis. Science 314, 130-133. doi: 10.1126/science. 1134108

Nölle, A., Zeug, A., Van Bergeijk, J., Tönges, L., Gerhard, R., Brinkmann, H., et al. (2011). The spinal muscular atrophy disease protein SMN is linked to the Rho-kinase pathway via profilin. Hum. Mol. Genet. 20, 4865-4878. doi: 10. 1093/hmg/ddr425

Oprea, G. E., Krober, S., McWhorter, M. L., Rossoll, W., Muller, S., Krawczak, M., et al. (2008). Plastin 3 is a protective modifier of autosomal recessive spinal muscular atrophy. Science 320, 524-527. doi: 10.1126/science.1155085

Panzeri, C., De Palma, C., Martinuzzi, A., Daga, A., De Polo, G., Bresolin, N., et al. (2006). The first ALS2 missense mutation associated with JPLS reveals new aspects of alsin biological function. Brain 129, 1710-1719. doi: 10 . 1093/brain/awl104

Pesaresi, M. G., Amori, I., Giorgi, C., Ferri, A., Fiorenzo, P., Gabanella, F., et al. (2011). Mitochondrial redox signalling by p66Shc mediates ALS-like disease through Racl inactivation. Hum. Mol. Genet. 20, 4196-4208. doi: 10. 1093/hmg/ddr347

Philips, T., and Robberecht, W. (2011). Neuroinflammation in amyotrophic lateral sclerosis: role of glial activation in motor neuron disease. Lancet Neurol. 10, 253 263. doi: 10.1016/s1474-4422(11)70015-1

Pollari, E., Goldsteins, G., Bart, G., Koistinaho, J., and Giniatullin, R. (2014). The role of oxidative stress in degeneration of the neuromuscular junction in amyotrophic lateral sclerosis. Front. Cell. Neurosci. 8:131. doi: 10.3389/fncel. 2014.00131

Rage, F., Boulisfane, N., Rihan, K., Neel, H., Gostan, T., Bertrand, E., et al. (2013). Genome-wide identification of mRNAs associated with the protein SMN whose depletion decreases their axonal localization. RNA 19, 1755-1766. doi: 10 . 1261/rna.040204.113

Renton, A. E., Chio, A., and Traynor, B. J. (2014). State of play in amyotrophic lateral sclerosis genetics. Nat. Neurosci. 17, 17-23. doi: 10.1038/nn.3584

Renton, A. E., Majounie, E., Waite, A., Simon-Sanchez, J., Rollinson, S., Gibbs, J. R., et al. (2011). A hexanucleotide repeat expansion in C9ORF72 is the cause of chromosome 9p21-linked ALS-FTD. Neuron 72, 257-268. doi: 10.1016/j. neuron.2011.09.010

Rizzo, F., Riboldi, G., Salani, S., Nizzardo, M., Simone, C., Corti, S., et al. (2014). Cellular therapy to target neuroinflammation in amyotrophic lateral sclerosis. Cell. Mol. Life Sci. 71, 999-1015. doi: 10.1007/s00018-013-1480-4

Rossoll, W., Jablonka, S., Andreassi, C., Kroning, A. K., Karle, K., Monani, U. R., et al. (2003). Smn, the spinal muscular atrophy-determining gene product, modulates axon growth and localization of beta-actin mRNA in growth cones of motoneurons. J. Cell Biol. 163, 801-812. doi: 10.1083/jcb. 200304128

Schrank, B., Gotz, R., Gunnersen, J. M., Ure, J. M., Toyka, K. V., Smith, A. G., et al. (1997). Inactivation of the survival motor neuron gene, a candidate gene for human spinal muscular atrophy, leads to massive cell death in early mouse embryos. Proc. Natl. Acad. Sci. U S A 94, 9920-9925. doi: 10.1073/pnas.94.18. 9920

Sorce, S., and Krause, K. H. (2009). NOX enzymes in the central nervous system: from signaling to disease. Antioxid. Redox Signal. 11, 2481-2504. doi: 10. 1089/ARS.2009.2578

Takata, M., Tanaka, H., Kimura, M., Nagahara, Y., Tanaka, K., Kawasaki, K., et al. (2013). Fasudil, a rho kinase inhibitor, limits motor neuron loss in experimental models of amyotrophic lateral sclerosis. Br. J. Pharmacol. 170, 341-351. doi: 10. 1111/bph. 12277

Tönges, L., Günther, R., Suhr, M., Jansen, J., Balck, A., Saal, K. A., et al. (2014). Rho kinase inhibition modulates microglia activation and improves survival in a model of amyotrophic lateral sclerosis. Glia 62, 217-232. doi: 10.1002/glia. 22601

Topp, J. D., Gray, N. W., Gerard, R. D., and Horazdovsky, B. F. (2004). Alsin is a Rab5 and Rac1 guanine nucleotide exchange factor. J. Biol. Chem. 279, 2461224623. doi: 10.1074/jbc.m313504200

Travaglione, S., Loizzo, S., Ballan, G., Fiorentini, C., and Fabbri, A. (2014). The E. coli CNF1 as a pioneering therapy for the central nervous system diseases. Toxins (Basel) 6, 270-282. doi: 10.3390/toxins6010270

Trumbull, K. A., McAllister, D., Gandelman, M. M., Fung, W. Y., Lew, T., Brennan, L., et al. (2012). Diapocynin and apocynin administration fails to significantly extend survival in G93A SOD1 ALS mice. Neurobiol. Dis. 45, 137-144. doi: 10. 1016/j.nbd.2011.07.015

Turner, B. J., Alfazema, N., Sheean, R. K., Sleigh, J. N., Davies, K. E., Horne, M. K., et al. (2014). Overexpression of survival motor neuron improves neuromuscular function and motor neuron survival in mutant SOD1 mice. Neurobiol. Aging 35, 906-915. doi: 10.1016/j.neurobiolaging.2013.09.030

van Bergeijk, J., Rydel-Konecke, K., Grothe, C., and Claus, P. (2007). The spinal muscular atrophy gene product regulates neurite outgrowth: importance of the C terminus. FASEB J. 21, 1492-1502. doi: 10.1096/fj.06-7136com

van Blitterswijk, M., Baker, M. C., Bieniek, K. F., Knopman, D. S., Josephs, K. A., Boeve, B., et al. (2013). Profilin-1 mutations are rare in patients with amyotrophic lateral sclerosis and frontotemporal dementia. Amyotroph. Lateral Scler. Frontotemporal Degener. 14, 463-469. doi: 10.3109/21678421.2013. 787630

Vance, C., Rogelj, B., Hortobagyi, T., De Vos, K. J., Nishimura, A. L., Sreedharan, J., et al. (2009). Mutations in FUS, an RNA processing protein, cause familial amyotrophic lateral sclerosis type 6. Science 323, 1208-1211. doi: 10.1126/science. 1165942

Wu, C. H., Fallini, C., Ticozzi, N., Keagle, P. J., Sapp, P. C., Piotrowska, K., et al. (2012). Mutations in the profilin 1 gene cause familial amyotrophic lateral sclerosis. Nature 488, 499-503. doi: 10.1038/nature1128

Wu, D. C., Re, D. B., Nagai, M., Ischiropoulos, H., and Przedborski, S. (2006). The inflammatory NADPH oxidase enzyme modulates motor neuron degeneration in amyotrophic lateral sclerosis mice. Proc. Natl. Acad. Sci. U S A 103, 12132 12137. doi: 10.1073/pnas.0603670103 
Yamazaki, T., Chen, S., Yu, Y., Yan, B., Haertlein, T. C., Carrasco, M. A., et al. (2012). FUS-SMN protein interactions link the motor neuron diseases ALS and SMA. Cell Rep. 2, 799-806. doi: 10.1016/j.celrep.2012.08.025

Yang, Y., Hentati, A., Deng, H. X., Dabbagh, O., Sasaki, T., Hirano, M., et al. (2001). The gene encoding alsin, a protein with three guanine-nucleotide exchange factor domains, is mutated in a form of recessive amyotrophic lateral sclerosis. Nat. Genet. 29, 160-165. doi: 10.1038/ng1001-160

Conflict of Interest Statement: The authors declare that the research was conducted in the absence of any commercial or financial relationships that could be construed as a potential conflict of interest.
Received: 14 July 2014; accepted: 22 August 2014; published online: 08 September 2014. Citation: D'Ambrosi N, Rossi S, Gerbino V and Cozzolino M (2014) Racl at the crossroad of actin dynamics and neuroinflammation in Amyotrophic Lateral Sclerosis. Front. Cell. Neurosci. 8:279. doi: 10.3389/fncel.2014.00279

This article was submitted to the journal Frontiers in Cellular Neuroscience.

Copyright (๑) 2014 D'Ambrosi, Rossi, Gerbino and Cozzolino. This is an open-access article distributed under the terms of the Creative Commons Attribution License (CC BY). The use, distribution or reproduction in other forums is permitted, provided the original author(s) or licensor are credited and that the original publication in this journal is cited, in accordance with accepted academic practice. No use, distribution or reproduction is permitted which does not comply with these terms. 